\title{
Frequency response analysis of dielectric materials in a parallel- plate transmission line
}

\author{
Aaron Don M. Africa, Gregory James Pe, Robert Ianny Roi Quijano \\ Department of Electronics and Communications Engineering, De La Salle University, Manila 1004, Philippines
}

\begin{tabular}{l}
\hline \hline Article Info \\
\hline Article history: \\
Received Feb 25, 2020 \\
Revised Jan 18, 2021 \\
Accepted Mar 15, 2021 \\
\hline
\end{tabular}

\section{Keywords:}

Dielectric constant

Dielectric material analysis

Frequency response

Parallel-plate transmission line

\begin{abstract}
A transmission line allows different frequencies to conduct alternating current (AC). They are a peculiar type of wire that allows signal transmission while making it resistant to external noises. A parallel-plate transmission line is a type of transmission line designed with two parallel plates with a dielectric sheet material in the center, as the name implies. The parallel-plate transmission lines are usually used for a miniature setup in which the line prevents the signal from losing power. However, the line's frequency response is a varying setup in which a change in a parameter can fully change the frequency response of the line, and in turn trigger inefficiency. With this, different factors such as the conductor, the size, and the dielectric material of the parallel-plate transmission line can affect its frequency response. Specifically, the analysis of the transmission would test the various frequency responses when the dielectric sheet content is varied. The researchers will carry out experiments on air, Teflon, plexiglass, and E type glass dielectrics.
\end{abstract}

This is an open access article under the CC BY-SA license.

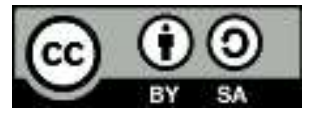

\section{Corresponding Author:}

Aaron Don M. Africa

Department of Electronics and Communications Engineering

De La Salle University, 2401 Taft Ave., Malate, Manila 1004, Philippines

Email: aaron.africa@dlsu.edu.ph

\section{INTRODUCTION}

Transmission lines are wire configurations normally used in transferring AC signals or pulses with frequencies in the microwave and radio wave spectrum. Although there exist many types of transmission lines such as twisted pair cables, optical fiber cables, and the likes, all serve the purpose of transmitting signals at long distances between two or more destinations. The general composition of a transmission line is made of two conductors that are commonly adjacent to a dielectric material. To put more bluntly, it is composed of two conductors sandwiching a conductor in some particular manner. Although a transmission line may come in many types, all of them share the aforementioned basic structure. The characteristic that makes each different, however, can be seen from the dielectric and conductor materials used in manufacturing them. They are also differentiated based on their shape, form, and intended purpose or fields of specialization. Relevantly, the parallel-plate transmission line modeled in this paper is a type of transmission line that consists of two parallel conductor planes separated by an insulating material. Common applications of such a transmission line are found in parallel-plate transmission line transformers [1] and as complements for frequency domain network analyzers [2]. Its geometry is also widely utilized in measuring the dielectric constants of printed circuit boards [3]-[4].

In this paper, the researchers strive to inspect the effects of the change in the dielectric material to the attenuation response of the parallel plate transmission line. The amplitude and phase attenuations introduced by the air, teflon, plexiglass, and E glass dielectric materials would be particularly investigated. 
Through this study, a thorough review of the aforementioned components will be performed that can further improve the material selection of insulators in transmission line designs. Depending on the application, this research can help inspect the best possible dielectric material performance-wise.

In recent decades, a variety of studies have been conducted concerning alterations in the materials used in manufacturing parallel-plate transmission lines and their respective effects [5]-[8]. This research differs from such papers such that it takes on a mathematical approach to exclusively analyze the behavior of the parallel-plate transmission line without considering external interferences. Also, observations will be performed through simulations in the Matlab software.

\section{BACKGROUND OF THE STUDY}

Transmission lines vary with different conductors, however, some transmission lines like the parallel plate transmission line vary their parameters with the adjustment of their dielectric material. Then it is important to note that when constructing any transmission line that requires a dielectric sheet, the different parameters of the dielectric must be kept at close inspection as it can significantly affect the results of the study. The usage of such transmission lines can also be made to be more efficient by properly implementing the different parameters of the dielectric sheet used. A paper by V. Astley, et al., [9] outlines the importance of utilizing proper dielectrics in their work in rectangular resonant cavities. Another study by D. M. French, et al., [10] outlines the effect of dielectrics in transmission lines by studying the losses of different dielectric materials. What both of these studies have in common is that it carefully determines not just the optimal transmission line but the optimal dielectric to utilize in each situation as well. Specifically, the research paper would want to analyze the frequency response of different dielectric materials when used with parallel transmission lines. In parallel plate transmission lines, the construction is simply two parallel plates that are placed next to each other. In the middle of these two plates is a dielectric sheet that assists the transmission line in signal transmission. The sheet in the middle is what the paper will be aiming to vary. By testing four different dielectric materials, Teflon, plexiglass, E-glass, and air. The study will then verify the individual strengths and weaknesses of each dielectric and will then be compared to each other. The paper aims to define the different advantages and disadvantages of using a specific dielectric substrate in terms of its frequency response in the amplitude to frequency plot and the frequency to phase plot.

\section{STATEMENT OF THE PROBLEM}

The amplitude and phase attenuation introduced by a transmission line is among the major causes of errors in the recovered signal. This is especially the case for long-distance transmissions where power losses and phase shifts become much more prevalent as the carrier travels farther. Per length of the transmission line and depending on its dielectric and conductor materials, a corresponding decibel of power is lost. Although this problem may be resolved through a signal repeater, its cost, installation, and maintenance make it a subjectively inefficient choice. As such, the researchers propose to analyze the frequency response of a parallel-plate transmission line using different insulating materials.

One of the aims of this paper is to establish the effects of a change in the dielectric material to the attenuation introduced by a parallel-plate transmission line. Consequently, the researchers will also inspect other possible alternatives for the dielectric materials. Insulating substances such as air, teflon, plexiglass, and e glass would be specifically investigated. A quantitative evaluation would be performed comparing the attenuation introduced by each material.

\section{SIGNIFICANCE OF THE STUDY}

Dielectrics are as much important as the conductors in parallel plate transmission lines or any other transmission line that requires dielectrics. Based on the work of R. Tamaru and F. Kuroki [11], the application of the dielectric material is well thought out and highly affects the results of their study. Another sample is the work by G. A. Kouzaev, et al., [12] wherein they investigate the different effects of a parallelplate guide wherein the dielectric used is specifically adjusted to fit the desired outputs of the paper. This research paper aims to evaluate the different frequency responses of the parallel-plate transmission line with varying dielectric materials. This is to determine the optimal material that is to be used in a specific scenario. However, it must be noted that the scope of the research will only be limited to the evaluation of the frequency response aspect of the dielectrics as they are integral in high speed printed circuit boards (PCBs). The research will include the frequency response analysis of air, teflon, plexiglass, and e type glass. The results of this paper will describe the varying advantages of each dielectric material and as well as their disadvantages with respect to the frequency response. 


\section{DESCRIPTION OF THE SYSTEM}

The evaluation of the frequency response analysis of the specific dielectrics will first include defining their parameters. Table 1 includes the different parameters of the dielectrics which include its relative permittivity and loss tangent. It must be noted that the specific dielectrics to be used are air, teflon, plexiglass, and e type glass.

Table 1. Properties of the given dielectric materials

\begin{tabular}{cccc}
\hline Name & Relative_Permittivity & Loss_Tangent & Frequency \\
\hline Air & 1 & 0 & $1.0000 \mathrm{e}+009$ \\
FR4 & 4.8000 & 0.0260 & $100.0000 \mathrm{e}+006$ \\
Teflon & 2.1000 & $2.0000 \mathrm{e}-04$ & $100.0000 \mathrm{e}+006$ \\
Foam & 1.0300 & $1.5000 \mathrm{e}-04$ & $50.0000 \mathrm{e}+006$ \\
Polystyrene & 2.5500 & $1.0000 \mathrm{e}-04$ & $100.0000 \mathrm{e}+006$ \\
Plexiglas & 2.5900 & 0.0068 & $10.0000 \mathrm{e}+009$ \\
Fused quartz & 3.7800 & $1.0000 \mathrm{e}-04$ & $10.0000 \mathrm{e}+009$ \\
E glass & 6.2200 & 0.0023 & $100.0000 \mathrm{e}+006$ \\
RO4725JXR & 2.5500 & 0.0022 & $2.5000 \mathrm{e}+009$ \\
RO4730JXR & 3 & 0.0023 & $2.5000 \mathrm{e}+009$ \\
TMM3 & 3.4500 & 0.0020 & $10.0000 \mathrm{e}+009$ \\
TMM4 & 4.7000 & 0.0020 & $10.0000 \mathrm{e}+009$ \\
TMM6 & 6.3000 & 0.0023 & $10.0000 \mathrm{e}+009$ \\
TMM10 & 9.8000 & 0.0022 & $10.0000 \mathrm{e}+009$ \\
TMM10i & 9.9000 & 0.0020 & $10.0000 \mathrm{e}+009$ \\
Taconic RF-35 & 3.5000 & 0.0018 & $1.9000 \mathrm{e}+009$ \\
\hline
\end{tabular}

\section{METHODOLOGY (WITH FLOWCHART)}

The flowchart of the research is shown in Figure 1. To obtain the frequency response of the parallelplate transmission line, the parameters of the transmission line model were first defined using default property values excluding the relative permittivity and loss tangent of the dielectric material; relative permittivity or Epsilon R, and the loss tangent of air, Teflon, plexiglass, and E glass are shown in Table 1. The ABCD and s-parameters of the model were then computed within the range of frequencies from 1-3 $\mathrm{GHz}$, different types of dielectric materials using the rfckt.parallel-plate(), analyze(), and then extract() commands. Thereafter, the transfer and rational function are computed which contain the computed and fitted data of the frequency response respectively. Lastly, such functions are plotted against the said frequency range using the plot command.

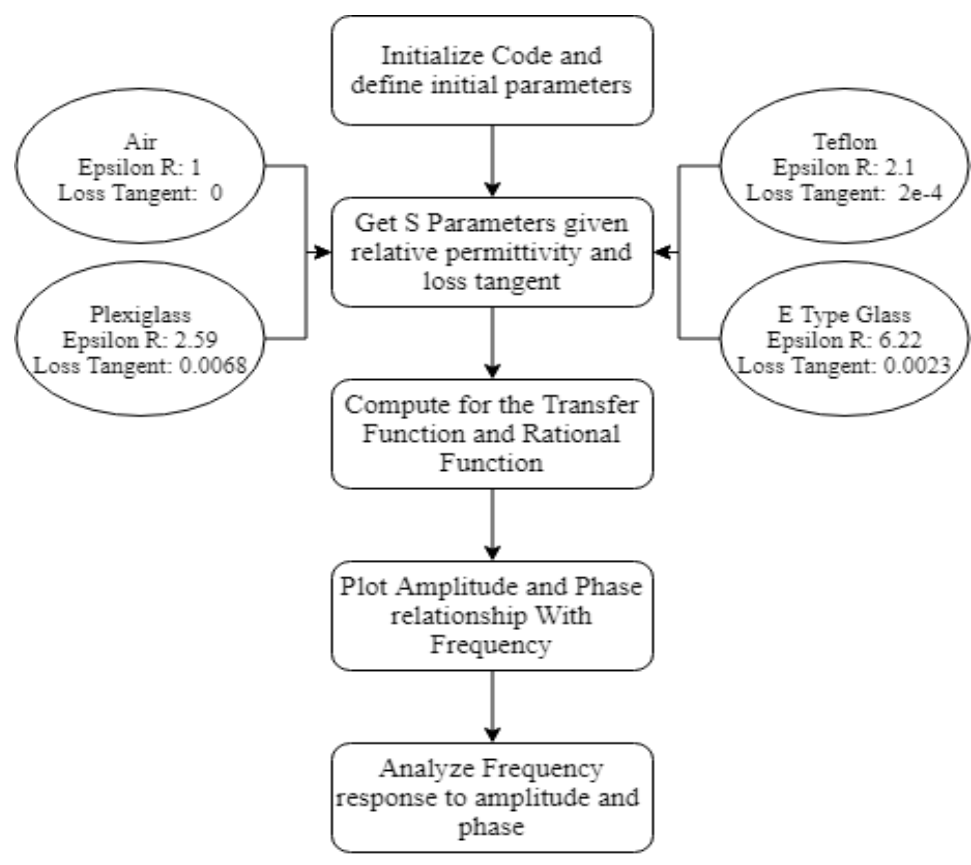

Figure 1. Flow diagram of the experiment 


\section{REVIEW OF THE RELATED LITERATURE}

In the research of Strickland, et al., [13], the dielectric constant and losses of fluid-impregnated films that are plastic in a parallel-plate transmission line were investigated at high frequencies. Their group aimed to develop a rep-rated high voltage pulse line that exhibited low loss and a high energy density with adequate reliability. As such, impregnating fluids such as water and propylene carbonate or ethylene glycol. were tested to determine their aptness in high voltage pulse forming lines. Relevantly, findings show that dielectric films with approximately 10 dielectric constant have dissipation factors within the range of 0.1 frequencies of $1 \mathrm{MHz}$.. On the other hand, films such as mylar and polypropylene, have factors more than one order of magnitude less even though they have low dielectric constants. The results of this research are important to the researchers since it establishes the fact that a change in the dielectric material has a significant effect on the amplitude attenuation of a parallel plate transmission line. This is evident in the fluid-impregnated plastic films used for the parallel-plate transmission line that exhibited different dissipation factors.

Using $\mathrm{NbN}$ and $\mathrm{YBa} 2 \mathrm{Cu} 3 \mathrm{O} 7-\mathrm{x}$ thin films, Tsuk, Shin, J. Oates, and D. Oates designed a nonlinear transmission line to investigate the nonlinear frequency response of striplines at high input power [14]. consequently, results show that inductances and resistances behaved nonlinearly for resonators with high $\mathrm{Q}$ factors. Aside from $\mathrm{NbN}$ and $\mathrm{YBCO}$ resonators, the hysteresis effect was also observed in the transmission lines at input power levels of $-6 \mathrm{dBm}$, allegedly due to nonlinear inductance. This paper is important to the researchers since it affirms that the frequency response of a transmission line, specifically a stripline, behaves nonlinearly and differently for most dielectric materials at high input power levels.

Voeten, vermeulen, pemen, and brussard proposed a transmission line transformer (TLT) design that utilized the geometry of the parallel-plate transmission line [15]. They aimed to limit the losses caused by reflections and the secondary mode to increase voltage gain effectively, which is allegedly best performed by the aforementioned transmission line. As such, their group examined the voltage amplification of the 8- and 4-line parallel-plate TLTs (PPTLS). Followingly, results show that they were able to achieve a near-ideal 1:1 ratio between the voltage gain and the number of lines in the PPTLT. This paper is crucial to the researchers since it contains and indicates relevant parameters for design considerations such as the breakdown voltage, flashover, and frequency response of the parallel-plate transmission line.

An alternative method for measuring the dielectric properties of thin films at microwave frequencies was proposed in the research of Song, et al., [16]. Their paper utilized the geometry of the parallel-plate transmission line in creating a waveguide structure to determine propagation and dielectric constants. In comparison to other forms of waveguides, their results show better accuracy and sensitivity in determining the aforementioned parameters. This paper is relevant to the researchers because it studies the electric field behavior of unconventional parallel-plate transmission lines; such a factor is known to exhibit an effect on the frequency response of such a transmission line.

In the study of Li, et al., [17], the saturation process of the multipactor of parallel-plate transmission lines at microwave frequencies was done in a simulation using particle in cell method. They performed their emulation by altering the weight values of microparticles until saturation is achieved at 2 hours of simulation time. Then, the critical frequency of saturation was determined alongside the noise and power loss introduced by the saturation current. Consequently, the results of their research show that the attenuation factor is directly proportional to the input power. In their designed model, an input power of $800 \mathrm{~W}$ causes a complete loss of the signal. This study is relevant to the researchers since it relates the input power to the amplitude attenuation of a parallel-plate transmission line at microwave frequencies. With regards to design considerations, the input power must be held constant throughout to not create any inconsistencies in their simulations.

In the research of Mechaik [18], the performance of coaxial cables and PCBs in transmitting data was evaluated using an accurate and efficient method of moments. particularly, the attenuation, dielectric, and conductive losses of such transmission lines were analyzed and modeled. Data procured in the research show that resistive losses dominate the total losses in PCBs and coaxial cables for frequencies up to $25 \mathrm{GHz}$. For frequencies higher than $25 \mathrm{GHz}$, dielectric losses become more prevalent than resistive losses. From these findings, Mechaik also established that all losses increase with frequency and vice-versa; there exists a major tradeoff when trying to transmit at higher bit rates. Though, to a certain extent, the total loss can be improved by utilizing dielectric and conductive materials with low losses. Although the aforementioned results may only apply for coaxial cables and PCBs, the findings of this paper are a good basis for the expected outcome of the frequency response analysis of the parallel-plate transmission line. To be exact, the researchers may expect that at frequencies around $25 \mathrm{GHz}$, the effects of different dielectric constants and losses become more apparent and distinct.

A mathematical description for the low-loss transmission attenuation constant in the presence of standing waves was modeled in the paper of Young, et al., [19]. Their research aimed to identify and correct 
errors in the measurement of attenuation constants within the microwave spectrum while taking into account resistive and dielectric losses. Relevantly, results show that measured attenuation constants using standing waves were inaccurate for transmission lines that are around a wavelength long. As the length is increased, however, these deviations become more negligible. Additionally, it was also observed that the attenuation constant is the same as that of the standing waves at transmission line lengths that are multiples of half the wavelength. This paper is important to the researchers since it affirms that there would be variations in the attenuation constant at different lengths of the transmission line in the presence of standing waves.

The research paper of [20] describes the different properties of materials that are dielectric. It is done by using the method of microstrip transmission line that is used to calculate the complex permittivity of a material. It must be known that a bandgap that is electromagnetic is used to bridge the microwave signals that pass theough the transmission line. The frequency used to measure the electrical properties was $2.4 \mathrm{GHz}$. This is then observed to further investigate the different magnitudes of the transmission response as well as the frequency information. The permittivity is then calculated using the observed responses, from the loss tangent the permittivity imaginary part is then calculated. With this, the researchers can extract different data with regards to the difference of dielectrics in a microstrip transmission line.

The study of M. Joler involves using Blumlein transmission lines that are folded in the creation of pulsed power compact drivers for a microwave power source [21]. The study outlines the different benefits of using high dielectric constant ceramics instead of using a Kapton as the dielectric for the modeling of the Blumlein transmission line. With this, the paper gives insight into how a dielectric can give different effects on the transmission and as well as a comparison between a ceramic and a kaption dielectric.

The study by X. Sun et al. outlines the importance of dielectric materials in high-speed printed circuit board (PCB) design [22]. The study states that to effectively extract the substrate properties, the truncation of the S-parameters of the transmission line is needed for the dielectric properties to be dominant. This is because the transmission line based material property extraction method requires the S-parameters to be a dominant factor. With this, the study then utilizes a binary search algorithm with a physics-based fitting method to find the truncation frequency in the least possible iterations.

The paper by O. Huber, et al., [23] is summary of the use of conductor-backed coplanar waveguide microstrip transmission lines in measuring constant and dielectric permittivity attenuation. The method must take into account several effects, such as frequency dependent, inductance, conductance, surface waves, and resistance, to determine the attenuation constant and dielectric permittivity [24]. The formula derived for any low loss transmission line from the compensation properties avoids extensive simulations that make it efficient. The validity of such a method is retrieved from RF-substrate materials for up to $110 \mathrm{GHz}$, with the results agreeing with different datasheets [25]. The research gives an idea as to how the different parameters of dielectrics such as attenuation constant and permittivity are determined.

\section{DATA AND RESULTS}

Figure 2 illustrates the amplitude attenuation response of the parallel-plate transmission line with different insulators between the frequency range of 1 to $3 \mathrm{GHz}$. On the other hand, Figures 3 to 6 shows the distinction between computed and fitted data for the air, teflon, plexiglass, and E glass dielectric material respectively.

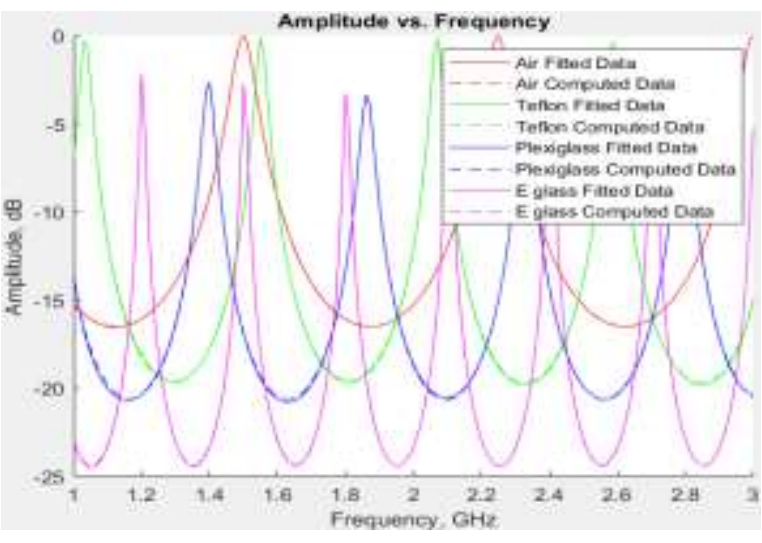

Figure 2. Frequency response analysis for the amplitude with corresponding computed and fitted data

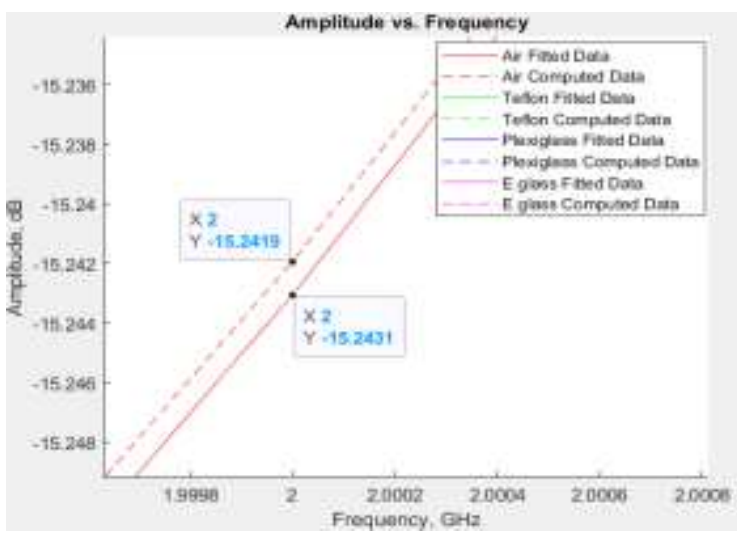

Figure 3. Amplitude attenuation distinctions for air dielectric 


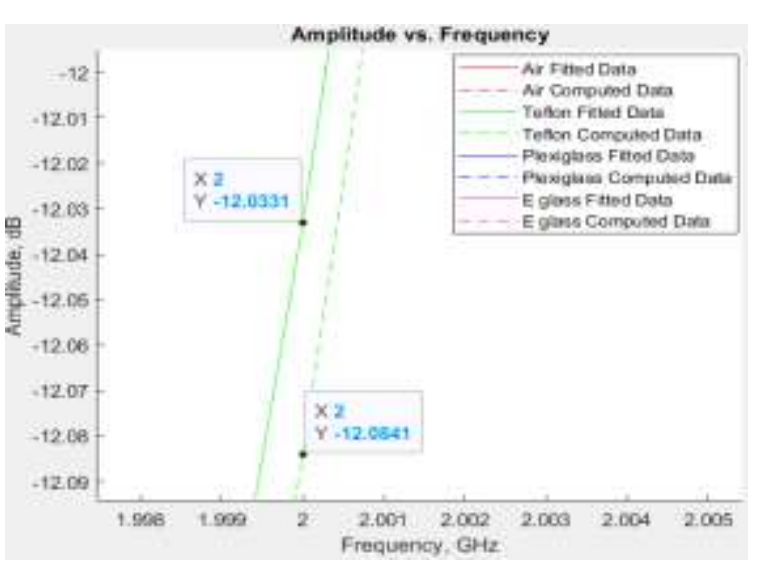

Figsure 4. Amplitude attenuation distinctions for teflon dielectric

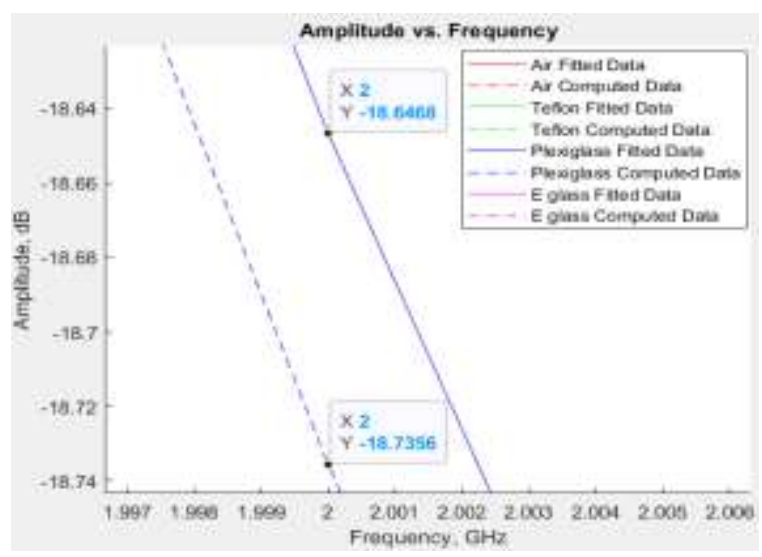

Figure 5. Amplitude attenuation distinctions for plexiglass dielectric

Figure 7 displays the phase angle attenuation response of the parallel-plate transmission line with different insulators between a frequency range of 1-3 GHz. On the other hand, Figures 8 to 9 illustrates the distinction between computed and fitted data for the air, teflon, plexiglass, and E glass dielectric material respectively.

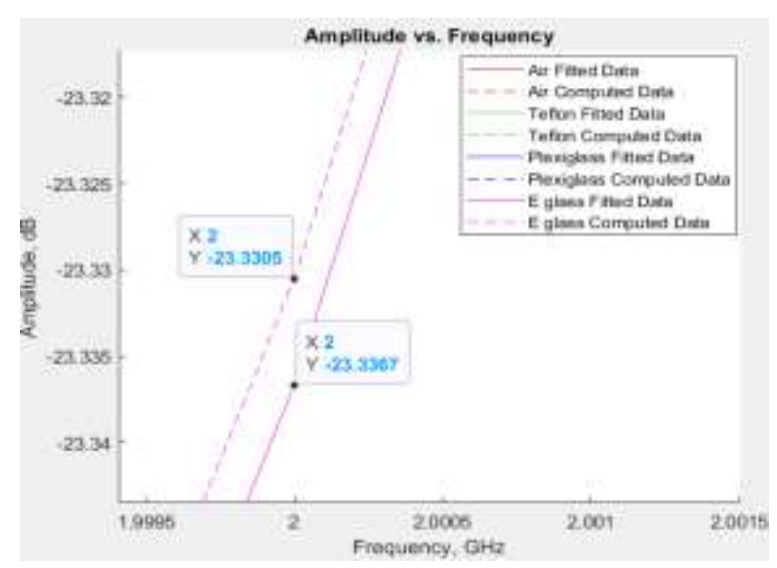

Figure 6. Amplitude attenuation distinctions for e glass dielectric

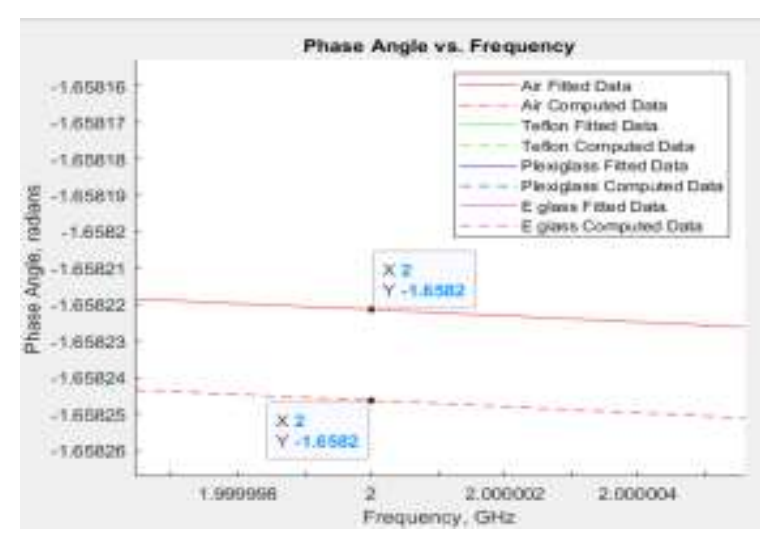

Figure 8. Phase angle attenuation distinctions for air dielectric

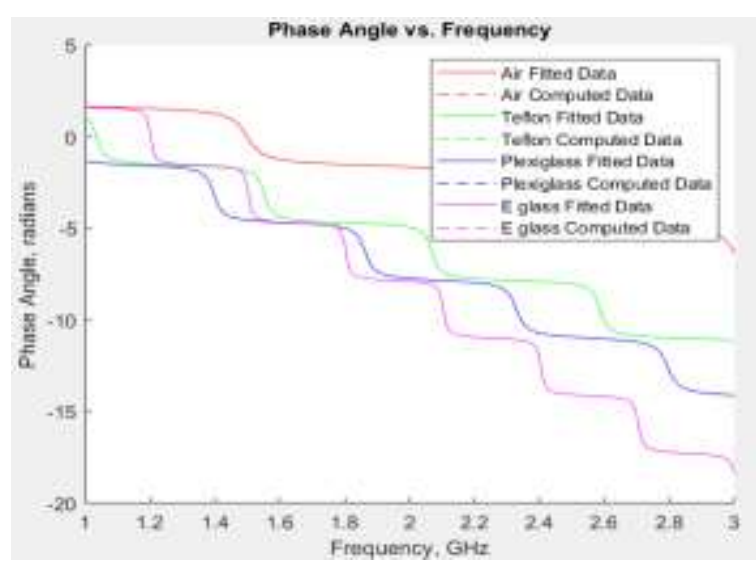

Figure 7. Frequency response analysis for the phase with corresponding computed and fitted data

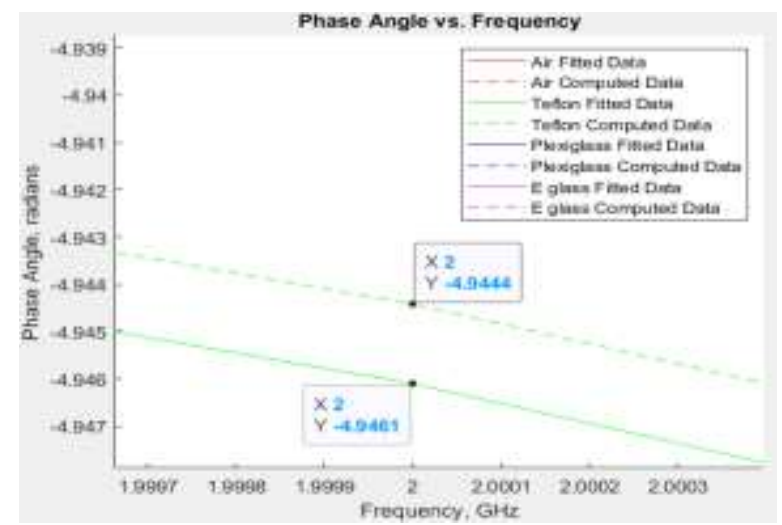

Figure 9. Phase angle attenuation distinctions for teflon dielectric 


\section{ANALYSIS OF DATA}

In Figure 2, it was apparent that the amplitude attenuation of the parallel-plate transmission line was non-linear for frequencies between 1 to $3 \mathrm{GHz}$. The amplitude response was seemingly, but not exactly, sinusoidal as it peaks and drops at certain intervals of frequencies. Within the specified frequency range, it was observed that the dielectric that introduced the least attenuation was air, followed by teflon, E glass, and then plexiglass. At $1.5 \mathrm{GHz}$, air exhibited a loss of $-0.00035761 \mathrm{~dB}$; $-0.11411 \mathrm{~dB}$ for teflon at $1.55 \mathrm{GHz}$; $2.2037 \mathrm{~dB}$ for E glass at $1.2 \mathrm{GHz}$; and $-2.6757 \mathrm{~dB}$ at $1.4 \mathrm{GHz}$. On the other hand, the material that exhibited the most attenuation was E glass, followed by plexiglass, teflon, and then air. At $1.35 \mathrm{GHz}$, E glass exhibited a loss of $-24.3912 \mathrm{~dB} ;-20.633 \mathrm{~dB}$ for plexiglass at $2.09 \mathrm{GHz} ;-19.6831 \mathrm{~dB}$ for teflon at $1.29 \mathrm{GHz}$; and $16.4836 \mathrm{~dB}$ for air at $1.87 \mathrm{GHz}$. From these values, a superficial trend could be observed such that the amplitude attenuation is directly proportional to the relative permittivity and loss tangent of the dielectric by some non-linear factor.

Figure 7 displays the relationship between the phase and frequency of different materials. The steepness of the material shows the phase difference per frequency, which means that the steeper the graph is the more the phase will vary. In Figure 7 the dielectric air causes the least phase difference of the bunch. The graph of air shows that it dips every $\sim 0.75 \mathrm{GHz}$ and stabilizes. it can also be seen that in Figure 8 the difference between the fitted and computed data is about 0.000015 radians. Teflon presents a steeper but better graph in terms of the phase difference with its dip occurring $\sim 0.5 \mathrm{GHz}$. Figure 9 presents the difference between the fitted and computed data of the Teflon dielectric, there is a considerable difference of around 0.0017 radians. The third dielectric material evaluated is the plexiglass and presents a dip at around $\sim 0.40 \mathrm{GHz}$ and reduces in time.

\section{CONCLUSION}

A Matlab-based algorithm was formulated to analyze the frequency response of a parallel-plate transmission line composed of different inner dielectric materials. The research aimed to establish the effects of a change in the dielectric material to the signal attenuation of the aforementioned transmission line and to inspect alternatives for its insulators. The amplitude and phase angle attenuation introduced by different values of relative permittivities and loss tangents were investigated and compared specifically that of air, teflon, plexiglass, and E glass. With regards to the methodology, a transmission line model was first generated in Matlab with default parameters excluding the loss tangent and relative permittivity of the dielectric. In graphing the frequency response, the s-parameters and transfer functions of the model were computed based on its ABCD parameters. The computed data and their fitted values were then plotted against the frequency range of 1 to $3 \mathrm{GHz}$. Concerning the amplitude response, results show that there exists a superficial trend between the amplitude attenuation and the loss tangent and relative permittivity of the dielectric; an increase in the latter causes an increase in the former and vice-versa. Within the specified frequency range, it was also observed that the least attenuation was achieved by air, followed by teflon, E glass, and plexiglass. The most attenuation, on the other hand, was introduced by E glass, followed by plexiglass, teflon, and air. Notably, there were negligible differences between the values of the computed and fitted amplitude responses of each material.

\section{REFERENCES}

[1] M. J. Lesha and F. J. Paoloni, "Impulse-optimised TEM horn antenna," Australian Journal of Electrical and Electronics Engineering, vol. 15, no. 1, pp. 31-37, 1995.

[2] V. P. McGinn, D. R. Mathis, and B. A. Smith "Low frequency RCS measurements with parallel plates," Proceedings of the Thirty-Sixth International Instrumentation Symposium, vol. 36, pp. 313-317, 1990.

[3] N. G. Paulter, "A fast and accurate method for measuring the dielectric constant of printed wiring board materials," IEEE Transactions on Components, Packaging, and Manufacturing Technology: Part C, vol. 19, no. 3, pp. 214225, 1996, doi: 10.1109/3476.558875.

[4] N. G. Paulter, "Accurate method for measuring the dielectric constant of printed wiring board materials," Circuit World, vol. 26, no. 1, pp. 27-32, 1999, doi: 10.1108/03056120010302197.

[5] C. J. Wu, "Propagation characteristics of a thin-film superconducting parallel-plate transmission line in the mixed state," Journal of Applied Physics, vol. 84, no. 6, pp. 3263-3266, 1998, doi.org/10.1063/1.368481.

[6] Africa, "Development of a wave propagation model in a lossy transmission line," International Journal of Emerging Trends in Engineering Research, vol. 8, no. 7, pp. 3351-3357, 2020, doi: 10.30534/ijeter/2020/76872020.

[7] Y. K. Cho, “Analysis of a narrow slit in a parallel-plate transmission line: E-polarisation case,” Electronics Letters, vol. 23, no. 21, pp. 1105-1106, 1987, doi: 10.1049/EL\%3A19870771. 
[8] L. Du, Y. Xie, and S. Wang, "Simulation computation and analytic modification of characteristic impedance of parallel-plate transmission line," Qiang Ji Guang Yu Lizishu/High Power Laser and Particle Beams, vol. 27, no. 8, 2015, doi: 10.11884/HPLPB201527.083201.

[9] V. Astley, B. McCracken, R. Mendis, and D. M. Mittleman, "Analysis of rectangular resonant cavities in terahertz parallel-plate waveguides," Opt. Lett., vol. 36, no. 8, p. 1452, 2011, doi: 10.1364/OL.36.001452.

[10] D. M. French, B. W. Hoff, S. Heidger, and D. Shiffler, "Dielectric nonlinear transmission line," IEEE Pulsed Power Conference (PPC), Jun. 2011, doi: 10.1109/PPC.2011.6191441.

[11] R. Tamaru and F. Kuroki, "High Permittivity Tape Transmission Line Embedded in Low Dielectric Support at Millimeter-Wave Frequencies," Korea-Japan Microwave Conference, Nov. 2007, doi: 10.1109/KJMW.2007.4402272.

[12] G. A. Kouzaev, M. J. Deen, and N. K. Nikolova, "A parallel-plate waveguide model of lossy microstrip lines," IEEE Microw. Wireless Compon. Lett., vol. 15, no. 1, pp. 27-29, 2005, doi: 10.1109/LMWC.2004.840970.

[13] N. C. Jaitly, A. Ramrus, Strickland, B.E., "High voltage pulsed performance of advanced dielectric materials," IEEE Conference Record of Power Modulator Symposium, pp. 373-380, 1988, doi: 10.1109/MODSYM.1988.26300.

[14] J. H. Oates, R. T. Shin, D. E. Oates, M. J. Tsuk, and P. P. Nguyen, "A nonlinear transmission line model for superconducting stripline resonators," IEEE Trans. Appl. Supercond., vol. 3, no. 1, pp. 17-22, Mar. 1993, doi: $10.1109 / 77.233413$

[15] S. J. Voeten, M. F. J. Vermeulen, G. J. H. Brussaard, and A. J. M. Pemen, "Parallel plate Transmission Line Transformer," IEEE Pulsed Power Conference (PPC), Jun. 2011, doi: 10.1109/PPC.2011.6191434.

[16] Ge Song, S. Follonier, A. Knoesen, and R. D. Miller, "Characterization of thin-film low-dielectric constant materials in the microwave range using on-wafer parallel-plate transmission lines," IEEE Microw. Guid. Wave Lett., vol. 10, no. 5, pp. 183-185, May 2000, doi: 10.1109/75.850371.

[17] Y. Li, Wen Qiang, Shu Lin and H. Wang, "Particle-in-cell simulation of saturation process of multipactor in parallel-plate transmission lines," IEEE International Vacuum Electronics Conference (IVEC), Beijing, pp. 1-2, 2015, doi: 10.1109/IVEC.2015.7224040.

[18] M. M. Mechaik, "Signal attenuation in transmission lines," Proceedings of the IEEE 2001. 2nd International Symposium on Quality Electronic Design, San Jose, CA, USA, pp. 191-196, 2001, doi: 10.1109/ISQED.2001.915226.

[19] J. Cole, R. J. Collier, and P. R. Young, "The Variation of the Attenuation Constant of Low-Loss Transmission Lines in the Presence of Standing Waves," IEEE Microwave and Wireless Components Letters, vol. 28, no. 8, pp. 639-641, Aug. 2018, doi: 10.1109/LMWC.2018.2848479.

[20] S. Subbaraj, V. S. Ramalingam, M. Kanagasabai, E. F. Sundarsingh, Y. P. Selvam, and S. Kingsley, "Electromagnetic Nondestructive Material Characterization of Dielectrics Using EBG Based Planar Transmission Line Sensor," IEEE Sensors J., vol. 16, no. 19, pp. 7081-7087, Oct. 2016, doi: 10.1109/JSEN.2016.2591320.

[21] M. Joler, "Study of High Dielectric Constant Materials for use in Compact Transmission Lines for HPM Drivers," BEAMS 2002: 14th International Conference on High-Power Particle Beams, 2002, doi: 10.1063/1.1530793.

[22] X. Sun et al., "An Efficient Approach to Find the Truncation Frequency for Transmission Line-Based Dielectric Material Property Extraction," IEEE Symposium on Electromagnetic Compatibility \& Signal/Power Integrity (EMCSI), Jul. 2018, doi: 10.1109/EMCSI.2018.8495409.

[23] O. Huber, T. Faseth, G. Magerl, and H. Arthaber, "Dielectric Characterization of RF-Printed Circuit Board Materials by Microstrip Transmission Lines and Conductor-Backed Coplanar Waveguides Up to $110 \mathrm{GHz}$," IEEE Trans. Microwave Theory Techn., vol. 66, no. 1, pp. 237-244, Jan. 2018, doi: 10.1109/TMTT.2017.2750152.

[24] H. Dong-Ho, Y. L. Li, R. A. Vieweg, T. G. Ruttan, and L. A. Polka, "Dielectric material characterization using rough surface transmission lines," 59th ARFTG Conference Digest, 2002, doi: 10.1109/ARFTGS.2002.1214679.

[25] E. Tanabe and W. T. Joines, "A nondestructive method for measuring the complex permittivity of dielectric materials at microwave frequencies using an open transmission line resonator," IEEE Trans. Instrum. Meas., IM-, vol. 25, no. 3, pp. 222-226, Sep. 1976, doi: 10.1109/TIM.1976.6312350.

\section{BIOGRAPHIES OF AUTHORS}

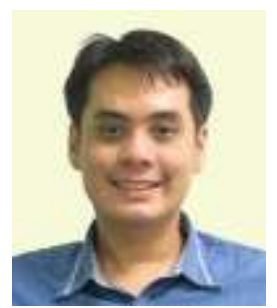

Aaron Don M. Africa works as a communications network engineer analyst. He's one of the field's most promising scholars. Communications Network Architecture, Expert Systems, Control Systems, Network Construction, and Optimization are his areas of specialization. He specializes in the optimization of communication systems to make them more functionally effective. 


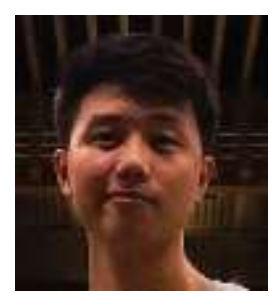

Gregory James Pe is a Filipino who hails from Manila. He is a De La Salle University student seeking a B.S. in Electronics and Communications Engineering (ECE). Transmission Line Analysis is one of his research interests, and he is a member of the Electronics and Communications Engineering Society (ECES).

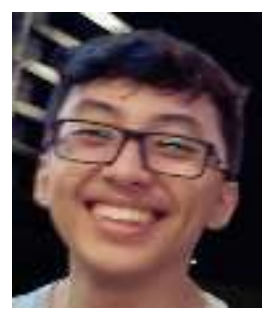

Robert Ianny Roi Quijano is a Filipino who hails from Manila. He is a De La Salle University student seeking a B.S. in Electronics and Communications Engineering (ECE). His academic interests include Signal Systems, and he is a member of the Electronics and Communications Engineering Society (ECES). 